\title{
Smart Cities, Privacy and the Pulverisation/Reconstruction of Individuals
}

\author{
Antoine Picon*
}

The smart city movement comprises strikingly different projects. One may for instance distinguish between top-down approaches, based on a neo-cybernetic, often technocratic inspiration, and bottom-up ones, which put the emphasis on citizens' initiatives and collaboration. Despite their differences, what these approaches have in common is the accent put on individuals. Because of their key role, it is the smartphone rather than the computer that epitomises the nature of the urban transition that we are facing.

Individuals appear as the main producers of data. Their movements in space, their online activities, their consumption of gas and electricity, their TV habits are tracked by innumerable administrations and corporations. Individuals may be considered as billions of events, the traces of which are stored in giant databases, before being mined for various purposes. In so doing, the ambition of the promoters of the smart movement is to make the city computable, as if these events were the real substance of urban life, and their traces the true matter of urban rationalisation.

In such a context, it is easy to be alarmed by the multiple invasions of individual privacy that have become all of a sudden possible, sometimes in almost sneaking ways. For instance, the new electricity smart meter that is currently installed in France, despite the protest of many users, makes it possible to detect, from the study of energy consumption profiles, what a family has actually watched on its main TV set hour after hour. Using geolocation, it is possible to know which doctors you are seeing and pry into some of your private medical issues.

The debate on privacy ranks among the most pressing political and legal issues that we have to address today. It might be however useful to frame it more precisely by observing that what is at stake may not be so much the knowledge of individuals in themselves as the way people are so to say pulverised into all sorts of elementary data or traces of events, in a manner reminiscent of the way the Internet uses packet switching to transmit information. Just like on the Internet, individuals are then partially reconstructed in order to give birth to 'profiles.' Corporations like the notorious GAFA', but also administrations generate profiles in order to recommend products, make de-

* Antoine Picon, G. Ware Travelstead Professor of the History of Architecture and Technology, Director of Research, Harvard Graduate School of Design. For correspondence: <apicon@gsd.harvard.edu>.

1 GAFA is an acronym referring to the Big Four technology companies Google, Amazon, Facebook, and Apple. 
cisions and elaborate strategies. Even more than the mere invasion of privacy, it is this process of reconstruction, which proves highly problematic. First, it tends to assimilate individuals to their profiles, something that raises in itself all kinds of questions. Are we reducible to subsets of the traces we leave in databases, as if these traces were like projections of stable identity? Profiles freeze our constantly-evolving features, delineating partial portraits which may be totally misleading. Above all, individuals who usually devote a lot of attention to their appearance and actions in the physical world or on social networks have absolutely no control over these profiles. In an age obsessed by individualism, the control of what is constructed from how we behave might represent a major right that we have to fight for. Computability should not be gained at the expense of such a right.

Beyond individuals, pulverisation followed by partial reconstruction appear as very general features of the digital age that we have entered. They possess an almost ontological and epistemological status, as if reality was reducible to traces, and as if beings and objects appeared always as combinations of these traces. Again, computability seems to require such a reduction. But the price to pay might be higher than what we imagine if this process is left unchecked. 